gr-qc/0610151

\title{
Thermodynamical properties of the Universe with dark energy
}

\author{
Yungui Gong* \\ CASPER, Department of Physics, Baylor University, Waco, TX 76798, USA \\ Bin Wang ${ }^{\dagger}$ \\ Department of Physics, Fudan University, Shanghai 200433, China \\ Anzhong Wang $\ddagger$ \\ CASPER, Department of Physics, Baylor University, Waco, TX 76798, USA
}

\begin{abstract}
We have investigated the thermodynamical properties of the Universe with dark energy. Adopting the usual assumption in deriving the constant co-moving entropy density that the physical volume and the temperature are independent, we observed some strange thermodynamical behaviors. However, these strange behaviors disappeared if we consider the realistic situation that the physical volume and the temperature of the Universe are related. Based on the well known correspondence between the Friedmann equation and the first law of thermodynamics of the apparent horizon, we argued that the apparent horizon is the physical horizon in dealing with thermodynamics problems. We have concentrated on the volume of the Universe within the apparent horizon and considered that the Universe is in thermal equilibrium with the Hawking temperature on the apparent horizon. For dark energy with $w \geq-1$, the holographic principle and the generalized second law are always respected.
\end{abstract}

\footnotetext{
*Electronic address: yungui gong@baylor.edu

${ }^{\dagger}$ Electronic address: wangb@fudan.edu.cn

${ }^{\ddagger}$ Electronic address: anzhong`wang@baylor.edu
} 


\section{INTRODUCTION}

Since the great discovery that the Universe is experiencing accelerated expansion driven by dark energy (DE) from the type Ia supernova (SN Ia) observations in 1998 [1], many works have been done in pursuit of understanding this spectacular phenomena. The later more accurate SN Ia data [2], together with the Wilkinson Microwave Anisotropy Probe data [3] and the Sloan Digital Sky Survey data [4] indicate that the Universe is almost spatially flat and further support the existence of DE which contributes about $72 \%$ of the matter content of the present universe within the framework of Einstein's general relativity. Despite the robust observational evidence for the existence of DE, DE is still a major puzzle of modern cosmology. Except knowing that DE has negative pressure, we know little about its theoretical nature and origin. In this work we would like to study the DE from thermodynamical considerations.

In the semiclassical quantum description of black hole physics, it was found that black holes emit Hawking radiation with a temperature proportional to their surface gravity at the event horizon and they have an entropy which is one quarter of the area of the event horizon in Planck unit [5]. The temperature, entropy and mass of black holes satisfy the first law of thermodynamics [6]. On the other hand, it was shown that the Einstein equation can be derived from the first law of thermodynamics by assuming the proportionality of entropy and the horizon area [7]. The Einstein equation for the nonlinear gravitational theory $f(R)$ was also derived from the first law of thermodynamics with some non-equilibrium corrections [8]. For a general static spherically symmetric space-time, Padmanabhan showed that the Einstein equation at the horizon gives the first law of thermodynamics on the horizon [9]. The study on the relation between the Einstein equation and the first law of thermodynamics has been generalized to the cosmological context where it was shown that the first law of thermodynamics on the apparent horizon $\tilde{r}_{A}$ can be derived from the Friedmann equation and vice versa if we take the Hawking temperature $T_{A}=1 / 2 \pi \tilde{r}_{A}$ and the entropy $S_{A}=\pi \tilde{r}_{A}^{2} / G$ on the apparent horizon [10]. Furthermore, the equivalence between the first law of thermodynamics and Friedmann equation was also found for gravity with GaussBonnet term and the Lovelock gravity theory [10, 11]. These results disclosed that there may be a deep relationship between the thermodynamics of the horizon and the Einstein equation, which might shed some light on the properties of DE. 
Motivated by the black hole thermodynamics, Bekenstein proposed a universal entropy bound for a weakly self-gravitating physical system in 1981 [12]. 't Hooft and Susskind subsequently built an influential holographic principle [13] relating the maximum number of degrees of freedom in a volume to its boundary surface area [14, 15]. The extension of the holographic principle to a general cosmological setting was first addressed by Fischler and Susskind [16] and subsequently got modified by many authors [17, 18]. The idea of holography is viewed as a real conceptual change in our thinking about gravity [19]. There have been some examples of applying the holographic principle to understand cosmological problems. It is interesting to note that holography implies a possible value of the cosmological constant in a large class of universes [20]. In an inhomogeneous cosmology holography was also realized as a useful tool to select physically acceptable models [21]. The idea of holography has further been applied to the study of inflation and gives possible upper limits to the number of e-folds [22]. Recently, holography has again been employed to investigate the DE [23] and it is expected that the holographic principle could serve as an effective way to help us understand the DE.

The Universe can be considered as a thermodynamical system. The thermodynamics in de Sitter space-time was first investigated by Gibbons and Hawking in [24]. In a spatially flat de Sitter space-time, the event horizon and the apparent horizon of the Universe coincide and there is only one cosmological horizon. It was found that the area of the cosmological horizon can be interpreted as the entropy or lack of information of the observer about the regions which he or she cannot see, and an observer with a particle detector will observe a background of thermal radiation coming from the cosmological horizon. The thermodynamical study of the Universe has been extended to the quasi-de Sitter space in [25, 26, 27]. When the apparent horizon and the event horizon of the Universe are different, it was found that the first law and the second law of thermodynamics hold on the apparent horizon, while break down if we consider the event horizon [27]. Due to this subtlety, we will concentrate our attention on the thermodynamical properties of the Universe enveloped by the apparent horizon. We will derive the temperature and entropy of the matter contents inside the apparent horizon from the first law of thermodynamics and discuss the holographic entropy bound and the generalized second law (GSL) of thermodynamics for the Universe with DE.

In the discussion of the thermodynamical properties of the matter contents, it is usually assumed that the physical volume and temperature of the Universe are independent and 
by using the integrability condition $\partial^{2} S / \partial T \partial V=\partial^{2} S / \partial V \partial T$ one has the constant comoving entropy density $\sigma$ and the relation $T s=\rho+p$ for the physical entropy density $s$. It follows that either the entropy or the temperature is negative for the phantom. Applying the constant co-moving entropy density to the first law of thermodynamics for DE with constant equation of state $w=p / \rho$, the thermodynamical properties of DE and the thermal spectral distribution were discussed in [28]. The thermodynamics of the DE with constant $w$ in the range $-1<w-1 / 3$ was also discussed in [29], and the thermodynamics of phantom with $w<-1$ was investigated in [30]. Some other discussions on this topic can be found in [31, 32]. However, in general, when we consider the thermal equilibrium state of the Universe, the temperature of the Universe is associated with the horizon, then the integrability condition cannot be used to derive the constancy of the co-moving entropy density from the first law of thermodynamics. Some assumptions on the temperature or entropy are needed to derive the thermodynamical properties of DE. With the requirement that the entropy of DE is bounded from above and the assumption that it could not increase faster than that of cosmic microwave background radiation, we will investigate the thermodynamical properties of DE in the thermal equilibrium universe. Employing the holographic entropy bound, we will also discuss the GSL for the Universe with DE. The study of GSL in a phantom dominated universe was carried out in [33]. We will address the thermodynamics of DE by considering the DE models with constant $w$ and the generalized Chaplygin gas (GCG) model which extrapolates between a dust and a cosmological constant [34].

The paper is organized as follows. In section II, we review the equivalence between Friedmann equation and the first law of thermodynamics of the apparent horizon. In section III, we discuss the thermodynamical consequence by assuming the physical volume and temperature are independent. In section IV, considering the physical volume is a function of the temperature, we investigate the thermodynamical properties for the DE with constant $w$ and the generalized Chaplygin gas. We discuss the GSL of thermodynamics for the Universe with DE in section $\mathrm{V}$. The summary and discussion will be presented in the last section VI,

\section{FIRST LAW OF THERMODYNAMICS ON THE APPARENT HORIZON}

For a spherically symmetric space-time with the metric $d s^{2}=g_{a b} d x^{a} d x^{b}+\tilde{r}^{2} d \Omega^{2}$, where

the unit spherical metric $d \Omega^{2}=d \theta^{2}+\sin ^{2} \theta d \phi^{2}$, we define the Misner-Sharp mass $M$ as 
[35, 36]

$$
g^{a b} \tilde{r}_{, a} \tilde{r}_{, b} \equiv f \equiv 1-\frac{2 G M}{\tilde{r}} .
$$

The mass $M$ defined in Eq. (1) was shown to be the active gravitational energy in the vacuum, small sphere, large sphere, Newtonian, test particle and relativistic limits [37]. The Einstein field equations tell us [35]

$$
M_{; a}=4 \pi \tilde{r}^{2}\left(T_{a}^{b}-\delta_{a}^{b} T_{c}^{c}\right) \tilde{r}_{; b}
$$

where the semicolon denotes covariant derivative with respect to the two dimensional metric $g_{a b}$. The apparent horizon is determined from $f=0$. We define the dynamic surface gravity at the apparent horizon as

$$
\kappa \equiv-\left.\frac{1}{2} \partial_{\tilde{r}}\right|_{f=0}
$$

So the Hawking temperature at the apparent horizon is $T=|\kappa| / 2 \pi$. The surface gravity $\kappa$ defined in Eq. (3) has the same form as the Newtonian surface gravity. In static black hole physics, the horizon is located at $f(\tilde{r})=1-2 G M / \tilde{r}=0$ which gives $\tilde{r}=2 G M$. The surface gravity is found to be $\kappa=-f^{\prime} / 2=-1 / 4 G M$ and the Hawking temperature is $T=1 / 8 \pi G M$. For the de Sitter universe, $f(\tilde{r})=1-H^{2} \tilde{r}^{2}$, the event horizon which coincides with the apparent horizon is $\tilde{r}_{A}=H^{-1}$. The surface gravity at the apparent (event) horizon is found to be $\kappa=-f^{\prime} / 2=\tilde{r}_{A}^{-1}$ and the Hawking temperature $T=1 / 2 \pi \tilde{r}_{A}$. For static spherically symmetric space-time, it was shown that Einstein equation at the apparent horizon $\tilde{r}_{i}$ gives the first law of thermodynamics of the apparent horizon with the identification of the temperature as $T=\left|f^{\prime}\left(\tilde{r}_{i}\right)\right| / 4 \pi$ and the entropy as $S=A / 4 G$, where $A=4 \pi \tilde{r}_{i}^{2}$ is the area of the horizon [9]. It was shown that the apparent horizon is a good boundary holding thermodynamical laws in the Universe driven by quintessence and phantom [27].

For the Friedmann-Robertson-Walker (FRW) metric, we have

$$
g_{a b}=\left(\begin{array}{cc}
-1 & 0 \\
0 & a^{2} /\left(1-k r^{2}\right)
\end{array}\right), \quad \tilde{r}=a r .
$$

So

$$
f=g^{a b} \tilde{r}_{, a} \tilde{r}_{, b}=1-\left(H^{2}+\frac{k}{a^{2}}\right) \tilde{r}^{2} .
$$

The apparent horizon is

$$
\tilde{r}_{A}=a r_{A}=\frac{1}{\sqrt{H^{2}+k / a^{2}}} .
$$


The surface gravity at the apparent horizon is $\kappa=-f^{\prime} / 2=1 / \tilde{r}_{A}$ and the Hawking temperature is

$$
T_{A}=\frac{1}{2 \pi \tilde{r}_{A}}
$$

By using the FRW metric (4), the definition of the Misner-Sharp mass (11), and the perfect fluid plus the cosmological constant for the matter, we get the Friedmann equations from the mass formulae (2),

$$
\begin{gathered}
H^{2}+\frac{k}{a^{2}}=\frac{8 \pi G}{3} \rho+\Lambda, \\
\dot{\rho}+3 H(\rho+p)=0, \\
\dot{H}-\frac{k}{a^{2}}=-4 \pi G(\rho+p) .
\end{gathered}
$$

Eq. (10) can be derived from Eqs. (8) and (9), so there are only two independent equations.

Combining Eqs. (6) and (10), we have

$$
\dot{\tilde{r}}_{A}=-\tilde{r}_{A}^{3} H\left(\dot{H}-k / a^{2}\right)=4 \pi G \tilde{r}_{A}^{3} H(\rho+p) .
$$

The energy flow through the apparent horizon is

$$
-d E=4 \pi \tilde{r}_{A}^{2} T_{\mu \nu} k^{\mu} k^{\nu} d t=4 \pi \tilde{r}_{A}^{3}(\rho+p) H d t
$$

where the (approximate) Killing vector or the (approximate) generators of the horizon, the future directed ingoing null vector field $k^{\mu}=(1,-H r, 0,0)$. Note that $d E=k^{a} M_{; a}$ with $k^{a} \tilde{r}_{; a}=0$. Therefore, we get the first law of thermodynamics of the apparent horizon

$$
T_{A} d S_{A}=\frac{1}{2 \pi \tilde{r}_{A}} \frac{d}{d t}\left(\frac{4 \pi \tilde{r}_{A}^{2}}{4 G}\right)=\dot{\tilde{r}}_{A} / G d t=-d E,
$$

if we define the entropy of the apparent horizon as $S_{A}=A / 4 G=\pi \tilde{r}_{A}^{2} / G$. It is clear that the first law of thermodynamics can be derived from Einstein equations. More interestingly, Einstein equations can be obtained from the first law. By using the first law of thermodynamics, we get

$$
\dot{\tilde{r}}_{A}=4 \pi G \tilde{r}_{A}^{3} H(\rho+p)=-\tilde{r}_{A}^{3} H\left(\dot{H}-k / a^{2}\right) .
$$

So

$$
\dot{H}-k / a^{2}=-4 \pi G(\rho+p) .
$$

Combining Eq. (15) with the energy conservation equation (9), we can derive Friedmann equation (8). In this derivation, there is also an integration constant which is set to be the 
cosmological constant. The derivation of Friedmann equation from thermodynamics was also shown for gravity with Gauss-Bonnet term and Lovelock gravity theories [11]. The equivalence between the Friedmann equation and the first law of thermodynamics of the apparent horizon for other gravitational theories, like the higher order gravity $f(R)$ and the Brans-Dicke theory, remains to be an open problem.

There are other general discussions on the first law of thermodynamics. In [37], the author derived a so called unified first law from the mass formulae (11),

$$
\nabla_{a} M=A \Psi_{a}+W \nabla_{a} V
$$

where the energy density $W=-\frac{1}{2} T_{a}^{a}$, the energy-supply vector $\Psi_{a}=T_{a}^{b} \partial_{b} \tilde{r}+W \partial_{a} \tilde{r}$, and the areal volume is determined by $\nabla V=A \nabla \tilde{r}$. The first term $A \Psi$ in the unified first law may be interpreted as an energy-supply term, analogous to the heat-supply term in the classical first law of thermodynamics, while the second term $W \nabla V$ may be interpreted as a work term. If we define the variation along the trapping horizon as $\delta M=z^{a} \nabla_{a} M$, where $z$ is a vector tangent to the trapping horizon and the normalization of $z$ is irrelevant, then it was shown that $A z^{a} \Psi_{a}=\kappa_{1} \delta A / 8 \pi$ and the unified first law becomes

$$
\delta M=\frac{\kappa_{1}}{8 \pi} \delta A+W \delta V
$$

where $\kappa_{1}=\square \tilde{r} / 2=M / \tilde{r}^{2}-4 \pi \tilde{r} W$. For cosmological models with FRW metric, $\kappa_{1} \neq \kappa$ and the above first law (17) is different from the one (13) derived from the Friedmann equation directly. Although the unified first law seems to be true for more general geometry with trapping horizon, our definitions of the surface gravity and the Hawking temperature (3) are appropriate for use in the cosmological context since the first law built reflects the requirement of the Einstein field equation.

Since at the apparent horizon, $f=0$, so the vector $\nabla_{a} f$ is normal to the apparent horizon, while the orthogonal vector $\zeta^{a}=\epsilon^{a b} \nabla_{b} f$ is tangent to it. If we define the variation at the apparent horizon as $\delta M=\zeta^{a} \nabla_{a} M$, then we have [26]

$$
\delta E=\zeta^{a} M_{; a}=\frac{M}{\tilde{r}_{A}^{2}} \tilde{r}_{A} \zeta^{a} \tilde{r}_{A ; a}=\frac{1}{4 \pi G \tilde{r}_{A}} \zeta^{a}\left(\pi \tilde{r}_{A}^{2}\right)_{; a}=\frac{T_{A}}{2} \delta S .
$$

In the above derivation, only the definitions of $M$ and $\tilde{r}_{A}$ are used. Although the formulae (18) looks in form like the first law of thermodynamics, it is different from Eq. (13). The physical meanings of $\delta E$ and $\delta S$ are different from $d E$ and $d S$ before. Further, Eq.(18) cannot reflect the dynamical property of the Universe. 
Thus, in the cosmological context, the apparent horizon is the physical proper size in discussing thermodynamics and the appropriate description of the first law on the apparent horizon is expressed in Eq. (13) with the definitions of the temperature in Eq. (17) and the entropy $S=\pi \tilde{r}_{A}^{2} / G$ on the apparent horizon. The first law for more general spherically symmetric space-times in other gravity theories needs further investigation.

\section{CONSTANT CO-MOVING ENTROPY DENSITY}

In an expanding universe, the first law of thermodynamics is

$$
T d S(T, V)=T d(s V)=d(\rho(T) V)+p(T) d V,
$$

where $s$ is the entropy density and $V$ is the physical volume. The energy density and the pressure are assumed to be functions of temperature only, and the volume $V$ and the temperature $T$ are usually assumed to be independent. The integrability condition $\partial^{2} S / \partial V \partial T=\partial^{2} S / \partial T \partial V$ relates the energy density and pressure

$$
\frac{d p}{d T}=\frac{\rho+p}{T} .
$$

For the cosmological constant $p=-\rho$, the first law implies $d S=d[V(\rho+p) / T]=0$. Substitute Eq. (20) into Eq. (19), we get

$$
s=\frac{\rho+p}{T}, \text { for } \rho \neq-p .
$$

For phantom field, we see that either the temperature or the entropy is negative. Combining Eqs. (19), (201) and (21), we get

$$
d s=\frac{d \rho}{T}=s \frac{d \rho}{\rho+p}, \text { for } \rho \neq-p .
$$

Therefore, once we are given the equation of state $p / \rho$, we can get the relationship between the entropy density $s$ and the energy density $\rho$, thence we can find the relationship between the temperature and the energy density. If we substitute the energy conservation equation (9) into Eq. (22), we get

$$
\sigma=s a^{3}=\text { constant }
$$

Therefore, the first law of thermodynamics combined with energy conservation tell us that the co-moving entropy density $\sigma$ is a constant. Note that the above results (21)-(23) are 
independent of the volume. They are derived based on the following assumptions: (1) The energy density and pressure are functions of temperature only; (2) The entropy is extensive so that we can define entropy density; (3) The volume and the temperature are independent.

In the discussion of the phantom thermodynamics, it was assumed that the temperature of $\mathrm{DE}$ is proportional to the horizon temperature, i.e., $T=b T_{A}$ [33]. For $b=1$, the $\mathrm{DE}$ is in thermal equilibrium with the horizon. We adopt this temperature of the Universe $T=b T_{A}$ to discuss the DE thermodynamical property. Substituting $T$ into Eq. (21), using Eqs. (10) and (23), we get

$$
s=\frac{\sigma}{a^{3}}=-\frac{\dot{H}-k / a^{2}}{2 b G\left(H^{2}+k / a^{2}\right)^{1 / 2}} .
$$

Considering the Friedmann equation (10), we see that for quintessence with $w>-1, s>0$, while for phantom with $w<-1, s<0$. For the flat universe $k=0$, the solution to Eq. (24) is

$$
a(t)=\left[2 b \sigma G\left(t-t_{*}\right)\right]^{1 / 3},
$$

where $t_{*}$ is an integration constant, or

$$
a(t)=\left[e^{3 d\left(t-t_{*}\right)}-\frac{2 b \sigma G}{3 d}\right]^{1 / 3},
$$

where $d \neq 0$ is an integration constant which is the asymptotical Hubble parameter. Only Eq. (26) gives the acceleration of the Universe driven by the DE. If $\sigma=0$, Eq. (26) becomes the cosmological constant solution. Substituting Eq. (26) into Friedmann equations (8)-(10), we get the DE equation of state

$$
p=-\rho\left[1-\frac{4 b \sigma G}{3 d} e^{-3 d\left(t-t_{*}\right)}\right] .
$$

Eq. (27) is the DE equation of state when the DE temperature $T=b T_{A}$. The DE is in thermal equilibrium with the apparent horizon when $b=1$. We see that when the DE is the cosmological constant, $\sigma=0$ and $p=-\rho$. If the DE persists with an equation of state different from that described in Eq. (27), it concludes that DE is not in thermal equilibrium with the apparent horizon in general. 


\section{A. Dark energy with constant $w$}

For a fluid with constant equation of state $p=w \rho$, the solution to the energy conservation equation (9) is $\rho_{w}=\rho_{w 0}\left(a_{0} / a\right)^{3(1+w)}$. From Eq. (22) we learn that

$$
\frac{s_{w}}{s_{w 0}}=\left(\frac{\rho_{w}}{\rho_{w 0}}\right)^{1 /(1+w)}=\left(\frac{a}{a_{0}}\right)^{-3},
$$

where the subscript "0" denotes the present time value. The entropy inside the apparent horizon is

$$
S_{w}=\frac{4}{3} \pi s_{w} \tilde{r}_{A}^{3}=S_{w 0}\left(\frac{a}{a_{0}}\right)^{-3}\left(\frac{\rho_{t}}{\rho_{t 0}}\right)^{-3 / 2},
$$

where $\rho_{t}$ is the total energy density and the Friedmann equation (8) was used to derive the last equation. During the radiation domination (RD), $\rho_{t}=\rho_{r} \sim a^{-4}, S_{R}=S_{R 0} \Omega_{r 0}^{-3 / 2}\left(a / a_{0}\right)^{3}$ and $S_{A}=\pi \tilde{r}_{A}^{2} / G=S_{A 0} \Omega_{r 0}^{-1}\left(a / a_{0}\right)^{4}$, where $\Omega_{r 0}=\rho_{r 0} / \rho_{t 0}$. During the matter domination (MD), $\rho_{t}=\rho_{m} \sim a^{-3}$, so $S_{R}=S_{R 0} \Omega_{m 0}^{-3 / 2}\left(a / a_{0}\right)^{3 / 2}$ and $S_{A}=S_{A 0} \Omega_{m 0}^{-1}\left(a / a_{0}\right)^{3}$. During the $w$-fluid domination, $\rho_{t}=\rho_{w} \sim a^{-(1+w)}$, then $S_{R}=S_{R 0} \Omega_{w 0}^{-3 / 2}\left(a / a_{0}\right)^{3(1+3 w) / 2}$ and $S_{A}=$ $S_{A 0} \Omega_{w 0}^{-1}\left(a / a_{0}\right)^{3(1+w)}$. During RD and MD eras, the entropy increases as the Universe expands, but when the DE dominates $w<-1 / 3$, the total entropy decreases as the Universe expands. It is easy to check that the entropy in each epoch of the evolution of the Universe is bounded by the apparent horizon entropy, once the entropy bound is satisfied at an earlier era. At very early epoch, the entropy bound is

$$
\frac{S_{R}}{S_{A}}=\frac{S_{R 0}}{S_{A 0}} \Omega_{r 0}^{-1 / 2}\left(\frac{a_{0}}{a}\right) \leq 1 .
$$

So $a / a_{0}=T_{\gamma 0} / T_{\gamma} \geq S_{R 0} \Omega_{r 0}^{-1 / 2} / S_{A 0} \sim 10^{-32}$ if we choose $H_{0}=72 \mathrm{Mpc}^{-1} \mathrm{~km} \mathrm{~s}^{-1}, S_{R 0} \sim 10^{88}$, $\Omega_{r 0} \sim 10^{-4}$ and $S_{A 0} \sim 10^{122}$, thus we have $T_{\gamma} \lesssim 10^{19} \mathrm{GeV}$. The relationship (28) between $s$ and $\rho$ may be used to provide other useful information, for example, if there is an energy bound, then we can get an entropy bound, which was used to argue the minimum mass of a primordial black hole in [38].

Substitute Eq. (28) into Eq. (21), we get

$$
\frac{T_{w}}{T_{w 0}}=\left(\frac{\rho_{w}}{\rho_{w 0}}\right)^{w /(1+w)}=\left(\frac{a}{a_{0}}\right)^{-3 w},
$$

where $T_{w 0}=(1+w) \rho_{w 0} / s_{w 0}$. For the radiation, $w=1 / 3$, we get the familiar relation $\rho \sim T^{4}$. For the DE, we see that it becomes hotter and hotter as the Universe expands. At present, the DE density $\rho_{w 0}$ is much greater than that of the radiation $\rho_{r 0}$, the DE temperature 
could be much higher than the current cosmic microwave background temperature unless $w$ is very close to -1 .

In [28], the authors find the spectral distribution $E^{1 / w} /[\exp (E / T) \pm 1]$. Assuming that the DE is massless and follows either the Bose-Einstein or Fermi-Dirac statistics, we get

$$
\rho=\frac{g}{2 \pi^{2}} \int_{0}^{\infty} \frac{E(P)}{\exp [E(P) / T] \pm 1} P^{2} d P \sim \frac{g}{6 \pi^{2} w} \int_{0}^{\infty} \frac{E^{1 / w}}{\exp [E(P) / T] \pm 1} d E
$$

where $P$ is the momentum and $g$ is the internal degrees of freedom. The number density is

$$
n=\frac{g}{2 \pi^{2}} \int_{0}^{\infty} \frac{P^{2} d P}{\exp (E / T) \pm 1} \sim \frac{g T^{1 / w}}{6 \pi^{2} w} \int_{0}^{\infty} \frac{u^{1 / w-1} d u}{e^{u} \pm 1}=n_{0}\left(\frac{T}{T_{0}}\right)^{1 / w} .
$$

Therefore, we get the dispersion relationship $E(P) \sim P^{3 w}$. For radiation, we recover the usual dispersion relationship $E=P$. For DE, we see that the greater the momentum, the smaller the energy. This is another strange property of DE. It could be that we cannot apply the above reasoning to the DE, i.e., the DE may not follow the usual statistics or it is not massless.

\section{B. The generalized Chaplygin gas}

For the GCG model, $p_{c}=-A / \rho_{c}^{\alpha}(\alpha>0)$, Eqs. (21) and (22) give us that

$$
\begin{gathered}
s_{c}^{\alpha+1}=C\left(\rho_{c}^{\alpha+1}-A\right) \\
T=C^{-1 /(1+\alpha)}\left(1-\frac{A}{\rho_{c}^{\alpha+1}}\right)^{\alpha / \alpha+1}
\end{gathered}
$$

The above relationships were derived in [32]. In terms of the variables $w_{c 0}$ and $\rho_{c 0}$, we can write $A=-w_{c 0} \rho_{c 0}^{\alpha+1}$. Eqs. (34) and (35) can be re-written as

$$
\begin{gathered}
\left(\frac{s_{c}}{s_{c 0}}\right)^{\alpha+1}=\frac{1}{1+w_{c 0}}\left[\left(\frac{\rho_{c}}{\rho_{c 0}}\right)^{1+\alpha}+w_{c 0}\right], \\
\rho_{c}=\rho_{c 0}\left[-\frac{1}{w_{c 0}}+\frac{1+w_{c 0}}{w_{c 0}}\left(\frac{T}{T_{0}}\right)^{(1+\alpha) / \alpha}\right]^{-1 /(1+\alpha)},
\end{gathered}
$$

where $T_{c 0}=\left(1+w_{c 0}\right)^{\alpha /(1+\alpha)} C^{-1 /(\alpha+1)}=\left(1+w_{c 0}\right) \rho_{c 0} / s_{c 0}$.

The solution to the energy conservation equation (9) is

$$
\rho_{c}=\rho_{c 0}\left[-w_{c 0}+\left(1+w_{c 0}\right)\left(\frac{a_{0}}{a}\right)^{3(1+\alpha)}\right]^{1 /(1+\alpha)} .
$$


This equation tells us that the GCG energy density decreases as the Universe expands and $\rho_{c} \rightarrow \rho_{c 0}\left(-w_{c 0}\right)^{1 /(1+\alpha)}$ as $a \rightarrow \infty$, i.e., the GCG extrapolates between a dust and a cosmological constant. From Eq. (37), we see that the temperature of the GCG starts from the maximum temperature $T_{m}=T_{0}\left(1+w_{c 0}\right)^{-\alpha /(1+\alpha)}$ and decreases to zero as the Universe expands. The temperature behavior of the GCG is different from that of the DE with constant $w$. The temperature of the GCG decreases instead of increasing. This tells us that the increase or decrease behavior of the temperature of the Universe dominated by DE is model dependent, it is not the general property associated with the DE.

\section{VOLUME AND TEMPERATURE INSIDE THE APPARENT HORIZON}

In cosmology, the physical volume is a function of the scale factor $a(t)$. Since the scale factor $a(t)$ relates to the temperature $T$ of the Universe, the volume $V$ should be a function of $T$. The first law (19) becomes

$$
(T s-\rho-p) d V=V(d \rho-T d s)
$$

The formula (21)-(23) are special solution to Eq. (39) and they are not valid in general. As discussed in section II], we should use the apparent horizon in the discussion of thermodynamics in cosmology [27]. For the radiation entropy, the previous results still hold. During $\mathrm{RD}$, the entropy contributed by relativistic particles is $S_{R} / S_{R 0}=\Omega_{r 0}^{-3 / 2}\left(a / a_{0}\right)^{3}$. During MD,

$S_{R} / S_{R 0}=\Omega_{m 0}^{-3 / 2}\left(a / a_{0}\right)^{3 / 2}$. Substituting $V=4 \pi \tilde{r}_{A}^{3} / 3$ into Eq. (39) and using Eq. (8), we get

$$
\begin{aligned}
T d S & =-\frac{2 \pi}{3}\left(\frac{8 \pi G}{3}\right)^{-3 / 2} \rho_{t}^{-5 / 2}\left[3(\rho+p) d \rho_{t}-2 \rho_{t} d \rho\right] \\
& =-\frac{2 \pi}{3}\left(\frac{8 \pi G}{3}\right)^{-3 / 2} \rho_{t}^{-5 / 2}\left(\rho_{t}+3 p_{t}\right) d \rho,
\end{aligned}
$$

where the second equality was obtained by using the energy conservation law. During DE domination, $T d S>0$ if $p<-\rho$ and $T d S \leq 0$ if $p \geq-\rho$.

\section{A. Dark energy in thermal equilibrium}

If we think that DE is in thermal equilibrium with the Hawking radiation of the apparent horizon, i.e., $T=T_{A}=1 / 2 \pi \tilde{r}_{A}$, then we get

$$
d S=-\frac{3}{16 G^{2}}\left(\rho_{t}+3 p_{t}\right) \rho_{t}^{-3} d \rho .
$$


For dark energy $p_{w}=w \rho_{w}$, the solution to Eq. (41) during RD is

$$
S_{w}=\frac{3(1+w)}{5-3 w} S_{A 0} \Omega_{r 0}^{-2} \Omega_{w 0}\left(\frac{a}{a_{0}}\right)^{5-3 w} .
$$

This is the DE entropy during RD period. During MD, the DE entropy can be obtained from Eq. (41) in the form

$$
S_{w}=S_{w 1}+\frac{1+w}{2(1-w)} S_{A 0} \Omega_{m 0}^{-2} \Omega_{w 0}\left(\frac{a}{a_{0}}\right)^{3(1-w)},
$$

where $S_{w 1}$ is an integration constant. We see that the entropy of the DE increases faster than those of the radiation and the apparent horizon during RD and MD. To require that the current DE entropy is negligible compared to the radiation entropy, we get

$$
\frac{1+w}{2(1-w)}\left(\frac{a_{t}}{a_{0}}\right)^{3 / 2-3 w} \ll \frac{S_{R 0} \Omega_{m 0}^{1 / 2}}{S_{A 0} \Omega_{w 0}} \sim 10^{-34},
$$

where $a_{t}$ is taken to be the end of the MD which is roughly the transition from deceleration to acceleration. The requirement of neglecting the DE entropy puts the constraint on the DE equation of state to be very close to -1 . This is consistent with recent observations. If the $\mathrm{DE}$ equation of state is different from -1 , then the $\mathrm{DE}$ entropy dominates the entropy components when the $\mathrm{DE}$ is in the thermal equilibrium with the apparent horizon. Comparing the DE entropy with the apparent horizon entropy

$$
\left.\frac{S_{w}}{S_{A}}\right|_{a \simeq a_{t}}=\frac{1+w}{2(1-w)} \frac{\Omega_{w 0}}{\Omega_{m 0}}\left(\frac{a_{t}}{a_{0}}\right)^{-3 w}<1,
$$

we find that the holographic bound does not put strict constraint on $w$ and it can be satisfied for general DE equation of state.

During DE domination, the radiation entropy contributed by relativistic particles becomes $S_{R} / S_{R 0}=\Omega_{w 0}^{-3 / 2}\left(a / a_{0}\right)^{3(1+3 w) / 2}$, the apparent horizon entropy becomes $S_{A} / S_{A 0}=$ $\Omega_{w 0}^{-1}\left(a / a_{0}\right)^{3(1+w)}$ and the solution to Eq. (41) gives the DE entropy

$$
S_{w}=S_{w i}+\frac{3}{16 G^{2}}(1+3 w) \rho_{w}^{-1}=S_{w i}+\frac{1+3 w}{2} S_{A 0} \Omega_{w 0}^{-1}\left(\frac{a}{a_{0}}\right)^{3(1+w)},
$$

where $S_{w i}$ is an integration constant. For DE with $w<-1 / 3$, the radiation entropy decreases while both the apparent horizon entropy and the absolute value of the DE entropy increase at the same rate. Eventually the radiation entropy will vanish and the DE entropy will become negative whose statistical meaning becomes hard to understand. The total entropy 
of the space-time is $S_{t}=S_{w}+S_{A}=S_{w i}+3(1+w) S_{A 0} \Omega_{w 0}^{-1}\left(a / a_{0}\right)^{3(1+w)} / 2$. Therefore the total entropy is positive if $w \geq-1$. To see the above results more clearly, we solve Eq. (41) numerically for $w=-0.9$ and the result is shown in Fig. 1, It is evident that $S_{w}$ increases faster than $S_{R}$ and $S_{w}>S_{R}$ during RD and MD, and $S_{w}$ is negative after MD. The entropy bound is always satisfied.

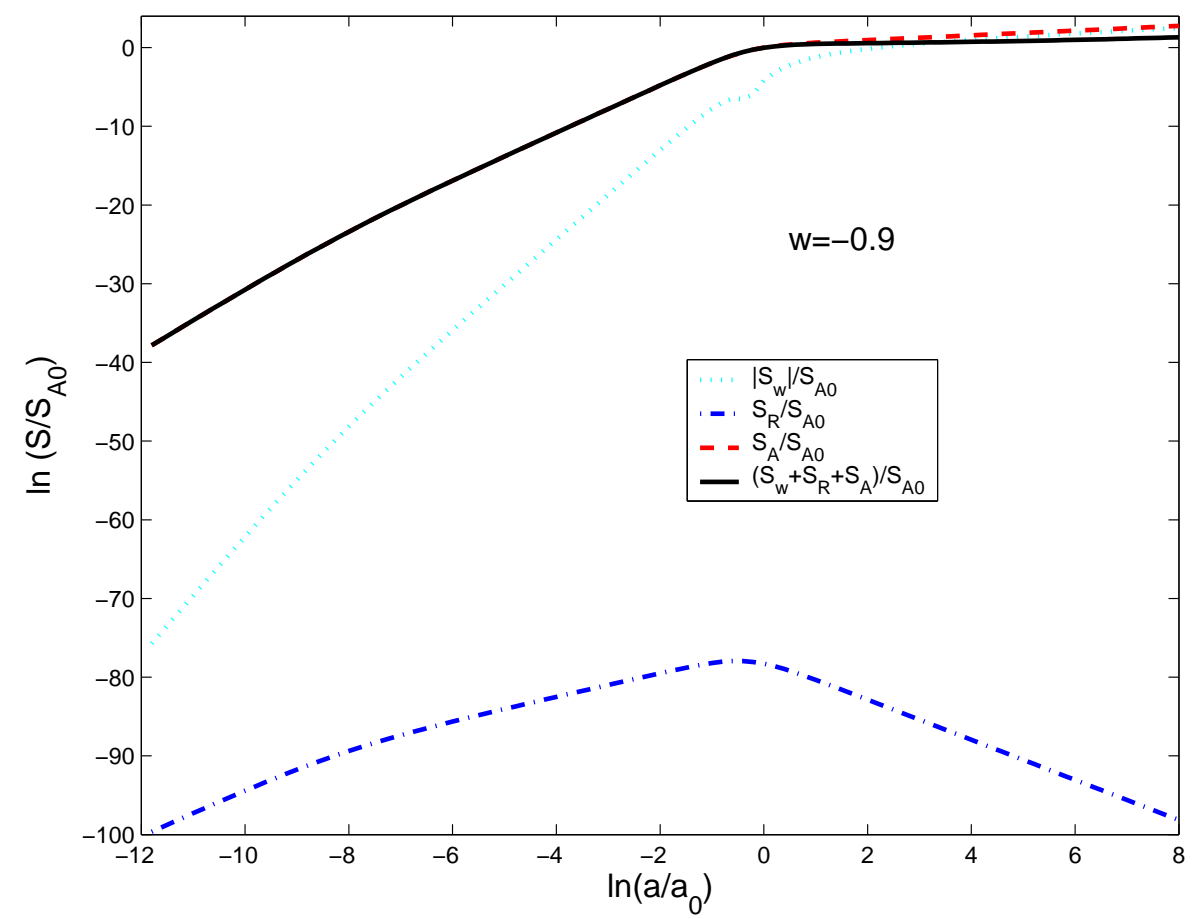

FIG. 1: The evolutions of $S_{w}, S_{R}$ and $S_{A}$. The dotted line is for $\left|S_{w}\right| / S_{A 0}$ (note that $S_{w}$ is negative after the MD), the dash-dot line is for $S_{R} / S_{A 0}$, the dashed line is for $S_{A} / S_{A 0}$, and the solid line is for the total entropy $\left(S_{A}+S_{R}+S_{w}\right) / S_{A 0}$.

For the GCG, Eq. (38) tells us that $\rho_{c}=\rho_{c 0}\left(1+w_{c 0}\right)^{1 /(1+\alpha)}\left(a_{0} / a\right)^{3}$ during RD and the solution to Eq. (41) is

$$
S_{c}=\frac{3}{5} S_{A 0} \Omega_{r 0}^{-2}\left(1+w_{c 0}\right)^{1 /(1+\alpha)}\left(\frac{a}{a_{0}}\right)^{5} .
$$

The entropy of the GCG increases faster than that of the radiation. 
During the epoch of the GCG domination, the solution to Eq. (41) is

$$
\begin{aligned}
S_{c} & =S_{c i}+\frac{1}{2} S_{A 0}\left(\frac{\rho_{c 0}}{\rho_{c}}\right)^{\alpha+2}\left[\left(\frac{\rho_{c}}{\rho_{c 0}}\right)^{\alpha+1}+\frac{3 w_{c 0}}{\alpha+2}\right] \\
& = \begin{cases}S_{c i}+\frac{1}{2} S_{A 0}\left(1+w_{c 0}\right)^{-1 /(1+\alpha)}\left(a / a_{0}\right)^{3}, & a \ll a_{0}, \\
\frac{\alpha-1}{2(2+\alpha)}\left(-w_{c 0}\right)^{-1 /(1+\alpha)} S_{A 0}, & a \gg a_{0},\end{cases}
\end{aligned}
$$

where $S_{c i}$ is an integration constant. It is easy to see that $S_{c 0}=S_{A 0}\left(\alpha+2+3 w_{c 0}\right) / 2(\alpha+2)$ and the holographic entropy bound is satisfied. If $\alpha \geq 1$, then the entropy is always non-negative and it increases first and then decreases. The apparent horizon entropy is

$$
\begin{aligned}
S_{A} & =S_{A 0}\left[-w_{c 0}+\left(1+w_{c 0}\right)\left(\frac{a}{a_{0}}\right)^{-3(1+\alpha)}\right]^{-1 /(1+\alpha)} \\
& = \begin{cases}\left(1+w_{c 0}\right)^{-1 /(1+\alpha)} S_{A 0}\left(a / a_{0}\right)^{3}, & a \ll a_{0}, \\
\left(-w_{c 0}\right)^{-1 /(1+\alpha)} S_{A 0}, & a \gg a_{0} .\end{cases}
\end{aligned}
$$

The entropy of the GCG is always smaller than that of the apparent horizon and the holographic bound is always respected. The radiation entropy is

$$
\begin{aligned}
S_{R} & =S_{R 0}\left(\frac{a}{a_{0}}\right)^{-3}\left[-w_{c 0}+\left(1+w_{c 0}\right)\left(\frac{a}{a_{0}}\right)^{-3(1+\alpha)}\right]^{-3 / 2(1+\alpha)} \\
& = \begin{cases}\left(1+w_{c 0}\right)^{-3 / 2(1+\alpha)} S_{r 0}\left(a / a_{0}\right)^{3 / 2}, & a \ll a_{0}, \\
\left(-w_{c 0}\right)^{-3 / 2(1+\alpha)} S_{r 0}\left(a / a_{0}\right)^{-3}, & a \gg a_{0} .\end{cases}
\end{aligned}
$$

The radiation entropy increases first and then decreases to zero. Since the entropy of the GCG increases faster than that of the radiation, the GCG entropy is greater than the radiation entropy at the present because $S_{c 0}>S_{r 0}$ and the holographic entropy bound is always respected. These results are evident from Fig. 2 with $w_{c 0}=-0.88$ and $\alpha=1.57$ [34]. So the GCG entropy is the dominate entropy if it is in thermal equilibrium with the apparent horizon.

\section{B. Dark energy with power law entropy}

Recall that the radiation temperature $T \sim a^{-1}$, the radiation entropy $S \sim T^{-3}$ during RD and $S \sim T^{-3 / 2}$ during MD. The relationship between the entropy and the temperature 


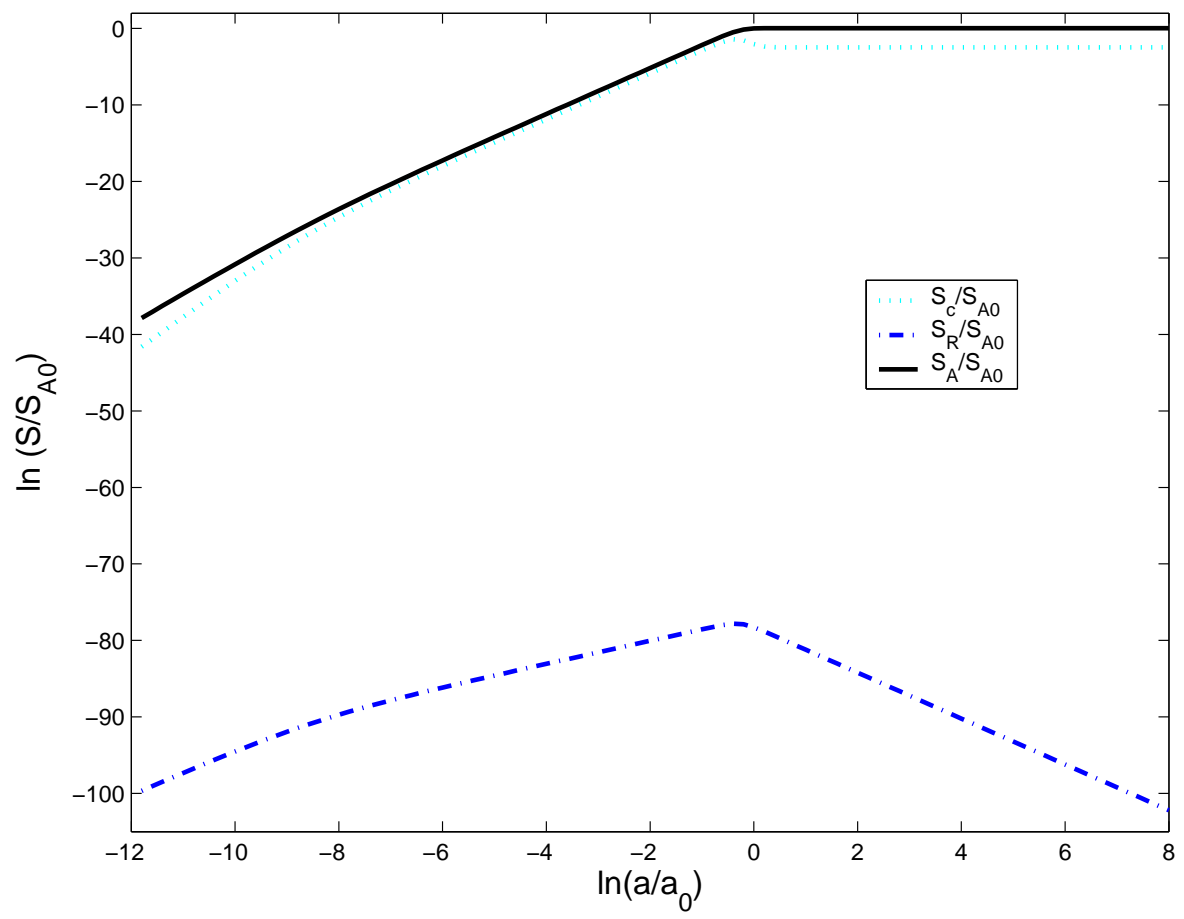

FIG. 2: The evolutions of $S_{c}, S_{R}$ and $S_{A}$ with $w_{c 0}=-0.88$ and $\alpha=1.57$. The dotted line is for $S_{c} / S_{A 0}$, the dash-dot line is for $S_{R} / S_{A 0}$, and the solid line is for $S_{A} / S_{A 0}$.

is a power law form. More generally, we assume that $S=\beta T^{\gamma}$, where the constants $\beta$ and $\gamma$ depend on the background, then Eq. (40) becomes

$$
\gamma \beta T^{\gamma} d T=-\frac{2 \pi}{3}\left(\frac{8 \pi G}{3}\right)^{-3 / 2}\left(\rho_{t}+3 p_{t}\right) \rho_{t}^{-5 / 2} d \rho .
$$

For the DE with $p=w \rho$, the solution to Eq. (51) during RD is

$$
\frac{\gamma_{w 1} \beta_{w 1}}{\gamma_{w 1}+1} T_{w}^{\gamma_{w 1}+1}=\frac{4 \pi(1+w)}{3(1-w)}\left(\frac{8 \pi G}{3}\right)^{-3 / 2} \rho_{r 0}^{-3 / 2} \rho_{w 0}\left(\frac{a}{a_{0}}\right)^{3(1-w)} .
$$

For radiation, $\gamma_{w 1}=-3$ and $T_{w} \sim a^{-1}$. If $\gamma_{w 1}<-1$ for $-1 / 3>w \geq-1$, then the DE temperature decreases and the entropy increases as the Universe expands. Since $S_{R} \sim a^{3}$ and $S_{w} \sim a^{3(1-w) \gamma_{w 1} /\left(1+\gamma_{w 1}\right)}>a^{3}$, the DE entropy increases faster than the radiation entropy. To make sure that the DE entropy is negligible compared to the radiation entropy, we require $0<\gamma_{w 1}<-1 / w$ so that $\gamma_{w 1} /\left(1+\gamma_{w 1}\right)>0$ and $3(1-w) \gamma_{w 1} /\left(1+\gamma_{w 1}\right)<3$ for $-1 / 3>w \geq-1$. Therefore the DE temperature and entropy both increase as the Universe expands. For $w<-1$, we need $-1<\gamma_{w 1}<0$ to keep the temperature positive. The phantom temperature increases and the entropy decreases as the Universe expands. However, both the temperature and the entropy of the phantom are positive. 
During MD, the solution to Eq. (51) is

$$
\frac{\gamma_{w 2} \beta_{w 2}}{\gamma_{w 2}+1} T_{w}^{\gamma_{w 2}+1}=C_{1}+\frac{4 \pi(1+w)}{3(1-2 w)}\left(\frac{8 \pi G}{3}\right)^{-3 / 2} \rho_{m 0}^{-3 / 2} \rho_{w 0}\left(\frac{a}{a_{0}}\right)^{3(1-2 w) / 2}
$$

where $C_{1}$ is an integration constant. If $\gamma_{w 2}<-1$ for $-1 / 3>w \geq-1$, then the DE entropy increases at a faster rate than that of the radiation as the Universe expands. So we require $0<\gamma_{w 2}<-1 / 2 w$ to keep $\gamma_{w 2} /\left(1+\gamma_{w 2}\right)>0$ and $3(1-2 w) \gamma_{w 2} / 2\left(1+\gamma_{w 2}\right)<3 / 2$. Again both the temperature and entropy of the DE with $w \geq-1$ increase as the Universe expands. For $w<-1$, we require $-1<\gamma_{w 2}<0$. The phantom temperature increase and the entropy decreases as the Universe expands.

During the DE domination, the solution to Eq. (51) is

$$
\frac{\gamma_{w 3} \beta_{w 3}}{\gamma_{w 3}+1} T_{w}^{\gamma_{w 3}+1}=C_{2}+\frac{4 \pi}{3}\left(\frac{8 \pi G}{3}\right)^{-3 / 2}(1+3 w)\left(\frac{a}{a_{0}}\right)^{3(1+w) / 2},
$$

where $C_{2}$ is an integration constant. Now we require that $-1<\gamma_{w 3}<0$ for both the quintessence and phantom to get a positive temperature. For $w \geq-1$, the temperature increases and the entropy decreases as the Universe expands. For $w<-1$, the temperature decreases and the entropy increases as the Universe expands. At late times, the entropy is

$$
\begin{aligned}
S_{w} & =\beta_{w 3}\left[\frac{4 \pi}{3 \beta_{w 3}}\left(\frac{8 \pi G}{3}\right)^{-3 / 2}(1+3 w) \frac{\gamma_{w 3}+1}{\gamma_{w 3}}\left(\frac{a}{a_{0}}\right)^{3(1+w) / 2}\right]^{\gamma_{w 3} /\left(\gamma_{w 3}+1\right)} \\
& \sim\left(\frac{a}{a_{0}}\right)^{3(1+w) \gamma_{w 3} / 2\left(1+\gamma_{w 3}\right)} .
\end{aligned}
$$

Even for the phantom, both the temperature and the entropy are positive. For $w \geq-1$, during the DE domination, the radiation entropy decreases as $a^{3(1+3 w) / 2}$, the DE entropy decreases as $a^{3(1+w) \gamma / 2(1+\gamma)}$ and the horizon entropy increases as $a^{3(1+w)}$, so the entropy bound will be respected. As we discussed earlier, the entropies of the radiation and DE with $w \geq-1$ increases slower than that of the apparent horizon, so the entropy bound is always satisfied. While for the phantom, $w<-1$, both the horizon entropy and the radiation entropy decrease and the phantom entropy increases, the entropy bound will be violated at late times.

For the GCG, the solution to Eq. (51) during RD is

$$
\frac{\gamma_{c 1} \beta_{c 1}}{\gamma_{c 1}+1} T_{c}^{\gamma_{c 1}+1}=\frac{4 \pi}{3}\left(\frac{8 \pi G}{3}\right)^{-3 / 2}\left(1+w_{c 0}\right)^{1 /(1+\alpha)} \rho_{r 0}^{-3 / 2} \rho_{c 0}\left(\frac{a}{a_{0}}\right)^{3} .
$$


So the temperature scales as $T_{c} \sim a^{3 /\left(1+\gamma_{c 1}\right)}$ and the entropy scales as $S_{c} \sim a^{3 \gamma_{c 1} /\left(1+\gamma_{c 1}\right)}$. If $\gamma_{c 1}<-1$, then the entropy increases faster than that of the radiation as the Universe expands. If $\gamma_{c 1}>0$, then both the temperature and the entropy increases and the entropy increases slower than that of the radiation as the Universe expands. During the GCG domination, the solution to Eq. (51) is

$$
\frac{\gamma_{c 2} \beta_{c 2}}{\gamma_{c 2}+1} T_{c}^{\gamma_{c 2}+1}=C_{3}+\frac{4 \pi}{3}\left(\frac{8 \pi G}{3}\right)^{-3 / 2} \rho_{c}^{-1 / 2}\left(\frac{\rho_{c 0}}{\rho_{c}}\right)^{\alpha+1}\left[\frac{-2 \alpha w_{c 0}}{3+2 \alpha}+\left(1+w_{c 0}\right)\left(\frac{a_{0}}{a}\right)^{3(1+\alpha)}\right] \text {, }
$$

where $C_{3}$ is an integration constant. At early times, $a \ll a_{0}$, the temperature scales as $T_{c} \sim a^{3 / 2\left(1+\gamma_{c 2}\right)}$ and the entropy scales as $S_{c} \sim a^{3 \gamma_{c 2} / 2\left(1+\gamma_{c 2}\right)}$. At late times, $a \gg a_{0}$, both the temperature and the entropy approach to a constant. To require that the DE entropy is negligible compared to that of radiation at least up to the present, we find that $\gamma_{c 2}>0$. So both the DE temperature and entropy increase first and then decrease and the DE entropy increases slower than that of the radiation.

\section{THE GENERALIZED SECOND LAW}

Now we proceed to discuss the GSL for the Universe with DE. The geometric entropy associated with the apparent horizon is $S_{A}=\pi \tilde{r}_{A}^{2} / G$. Using Eq. (11), we get

$$
\dot{S}_{A}=3 S_{A} H \frac{\rho_{t}+p_{t}}{\rho_{t}} .
$$

As long as $\rho_{t}+p_{t} \geq 0, \dot{S}_{A} \geq 0$, while for the phantom domination, $\dot{S}_{A}<0$.

\section{A. Constant co-moving entropy density}

The radiation entropy contributed by the relativistic particles inside the apparent horizon is $S=4 \pi s \tilde{r}_{A}^{3} / 3$, its time derivative reads

$$
\dot{S}=3 S H \frac{\rho_{t}+3 p_{t}}{2 \rho_{t}} .
$$

In the radiation or matter dominated eras, $S$ increases as the Universe expands if $S>0$, so the GSL is satisfied. The violation of the GSL is only possible when DE dominates. During the DE domination, the variation of the total entropy in the Universe is expressed as

$$
\dot{S}+\dot{S}_{A}=3 S H \frac{\rho_{t}+3 p_{t}}{2 \rho_{t}}+3 S_{A} H \frac{\rho_{t}+p_{t}}{\rho_{t}} .
$$


During RD and MD eras, the radiation entropy increases slower than that of the apparent horizon, so the entropy bound $S<S_{A}$ is always satisfied. Considering the equation of state in the RD and MD epoches, Eq. (60) is always positive so that the GSL is protected during $\mathrm{RD}$ and MD eras. When the DE starts to dominate, $S$ decreases as the Universe expands while $S_{A}$ keeps on increasing for $w>-1$, so in the far future $S \ll S_{A}$ for $w>-1$. Since $\left(\rho_{t}+3 p_{t}\right) / 2 \rho_{t}$ has the same order of magnitude as $\left(\rho_{t}+p_{t}\right) / \rho_{t}$, the GSL can be preserved for $w>-1$. To be more explicit, we take the DE model with constant $w$ as an example. In this case, Eq. (60) becomes

$$
\dot{S}+\dot{S}_{A}=3 H\left[\frac{1+3 w}{2} S_{0}\left(\frac{a}{a_{0}}\right)^{3(1+3 w) / 2}+(1+w) S_{A 0}\left(\frac{a}{a_{0}}\right)^{3(1+w)}\right] .
$$

For $w>-1,|(1+3 w) / 2| S_{0}<(1+w) S_{A 0}$ and the first term in the right hand side decreases and the second term increases, so the GSL is always satisfied.

For the phantom with $w<-1$, from the discussion in Sec. IIIA, we learnt that the temperature $T<0$ while the entropy $S>0$. The GSL is violated since $\dot{S}_{A}<0$ and $\dot{S}<0$ during the phantom domination. This problem can be attributed to the negative temperature deduced in the formalism where the volume and the temperature are assumed to be independent. In [33], by identifying the temperature to the apparent horizon temperature, $T>0$ and $S<0$, it was shown that the GSL may be satisfied.

\section{B. Volume as a function of Temperature}

Now we study the GSL by considering the physical volume and the temperature are dependent and taking the temperature of the Universe to be equal to the apparent horizon temperature. From Eq. (40), we see that $\dot{S} \geq 0(<0)$ if $(\rho+p) / T \geq 0(<0)$ during RD and MD, and $\dot{S} \leq 0(>0)$ if $(\rho+p) / T \geq 0(<0)$ during the DE domination. During the phantom domination, $\dot{S}_{A}<0$, so we must require $T>0$ to protect the GSL.

If $T=T_{A}$, Eq. (41) tells us that

$$
\dot{S}=\frac{9}{16 G^{2}}\left(\rho_{t}+3 p_{t}\right)(\rho+p) \rho_{t}^{-3} H .
$$

Combining Eqs. (58) and (62), we get

$$
\dot{S}_{t}+\dot{S}_{A}=\frac{27}{16 G^{2}} H \rho_{t}^{-3}\left(\rho_{t}+p_{t}\right)^{2} \geq 0
$$


for the total entropy of the Universe and the apparent horizon entropy. Therefore, the GSL is always satisfied even for the phantom.

For the case of $S=\beta T^{\gamma}$, we find that the entropies all increase during RD and MD. Only when the DE dominates, the entropies of the radiation and DE with $w>-1$ decrease. So we discuss the GSL during the DE domination. All the entropies have the power law

dependence on the scale factor $a$, so $\dot{S} \sim S H$ with an order one proportionality factor. Since $S_{R} \ll S_{A}$ and $S_{w} \ll S_{A}$, so the GSL is always respected.

\section{CONCLUSIONS AND DISCUSSION}

In this paper we have studied the thermodynamical properties of the Universe with DE. Adopting the usual assumption in deriving the constant co-moving entropy density that the physical volume and the temperature are independent, we studied the thermodynamics of the Universe in the radiation, matter and DE dominated periods. During RD and MD epoches, the entropy of the Universe contributed by the relativistic particles increase with the expansion, while in the DE dominated era, the entropy remains positive but decreases as the Universe expands. In all these processes, the entropy within the Universe is bounded by the geometric entropy associated with the apparent horizon. The holographic principle is respected. The requirement of the holography at early epoch gives reasonable energy scale. We have found some strange behaviors of the DE based on the assumption that the physical volume and the temperature are independent. For the DE with constant $w$, we have observed the strange dispersion relation $E \sim P^{3 w}$. For the phantom, we saw that either the temperature or the entropy could be negative. For the DE with constant equation of state $w>-1$, the temperature increases as the Universe expands. We have also extended our discussion to the GCG and found that its temperature decreases as the Universe expands, which is different from the DE with constant $w$. Thus the strange behavior on the temperature as observed is not the general property of the DE, it looks model dependent.

We have also considered the realistic situation that the physical volume and the temperature of the Universe are related. We have concentrated on the volume within the apparent horizon. The apparent horizon is important for the study of cosmology, since on the apparent horizon there is the well known correspondence between the first law of thermodynamics and Einstein equation. On the other hand it has been found that the apparent horizon is 
a good boundary for keeping thermodynamical laws [27]. Considering that the Universe is in thermal equilibrium, $T=T_{A}$, we have studied the entropy of the DE in the radiation, matter and DE dominated eras. We found the DE entropy increases during the RD and MD eras while decreases during the DE dominated era. In all epoches, the DE entropy is bounded by the holographic entropy on the apparent horizon. Requiring that at the present epoch, the DE entropy is negligible compared to the radiation entropy, we can limit the DE equation of state to be very close to -1 , which is consistent with observations. We found that both temperature and entropy can be positive for the DE including the phantom.

We have also investigated the GSL in the Universe with DE. By assuming the physical volume independent of the temperature, we have found that the GSL is protected for the DE with $w>-1$. Considering the realistic case that the physical volume and the temperature are related, and the DE inside the Universe is in thermal equilibrium with the boundary by the apparent horizon, the GSL is proved to be always satisfied.

The results tell us that in studying the thermodynamics of the Universe with DE, it is more appropriate to consider the case that the physical volume and the temperature are related. This could give us more reasonable results on the thermodynamical quantities in the Universe with DE.

\section{Acknowledgments}

YG is supported by Baylor University, NNSFC under grant No. 10447008 and 10605042 , CMEC under grant No. KJ060502 and SRF for ROCS, State Education Ministry. BW 's work was partially supported by NNSF of China, Ministry of Education of China and Shanghai Education Commission.

[1] A.G. Riess et al., Astron. J. 116 (1998) 1009; S. Perlmutter et al., Astrophy. J. 517 (1999) 565.

[2] A.G. Riess et al., Astrophy. J. 607 (2004) 665; P. Astier et al., Astron. and Astrophys. 447 (2006) 31-48.

[3] C.L. Bennett et al., Astrophys. J. Supp. Ser. 148 (2003) 1; D.N. Spergel et al., astro-ph/0603449 
[4] D.J. Eisenstein et al., Astrophys. J. 633 (2005) 560.

[5] S.W. Hawking, Commun. Math. Phys. 43 (1975) 199.

[6] J.M. Bardeen, B. Carter and S.W. Hawking, Commun. Math. Phys. 31 (1973) 161.

[7] T. Jacobson, Phys. Rev. Lett. 75 (1995) 1260.

[8] C. Eling, R. Guedens and T. Jacobson, Phys. Rev. Lett. 96 (2006) 121301.

[9] T. Padmanabhan, Class. Quantum Grav. 19 (2002) 5387.

[10] R.G. Cai and S.P. Kim, J. High Energy Phys. JHEP02(2005)050, hep-th/0501055.

[11] M. Akbar and R.G. Cai, Phys. Lett. B 635 (2006) 7; hep-th/0609128; A. Paranjape, S. Sarkar and T. Padmanabhan, Phys. Rev. D 74 (2006) 104015, hep-th/0607240.

[12] J.D. Bekenstein, Phys. Rev. D 23 (1981) 287.

[13] G. 't Hooft, Salam-festschrifft: a collection of talks, ed Aly A et al (World Scientific, Sigapore) P 284, 1993; L. Susskind, J. Math. Phys. 36 (1995) 6377.

[14] R. Bousso, J. High Energy Phys. JHEP07(1999)004, hep-th/9905177; Rev. Mod. Phys. 74 (2002) 825

[15] J.M. Maldacena, Adv. Theor. Math. Phys. 2 (1998) 231; E. Witten, Adv. Theor. Math. Phys. $2(1998) 253$

[16] W. Fischler and L. Susskind, hep-th/9806039;

[17] N. Kaloper and A. Linder, Phys. Rev. D 60 (1999) 103509; R. Easther and D.A. Lowe, Phys. Rev. Lett. 82 (1999) 4967; R. Brustein, Phys. Rev. Lett. 84 (2000) 2072; R. Brustein and G. Veneziano, Phys. Rev. Lett. 84 (2000) 5695; R. Bousso, J. High Energy Phys. JHEP06(1999)028, hep-th/9906022; ibid, Class. Quan. Grav. 17 (2000) 997; B. Wang and E. Abdalla, Phys. Lett. B 466 (1999) 122; ibid, 471 (2000) 346; B. Wang, E. Abdalla and R. K. Su, Phys. Lett. B 503 (2001) 394; G. Veneziano, Phys. Lett. B 454 (1999) 22; I. Savonijie and E. Verlinde, Phys. Lett. B 507 (2001) 305; B. Wang, E. Abdalla and R.-K. Su, Mod. Phys. Lett. A 17 (2002) 23; S. Nojiri and S. D. Odintsov, Int. J. Mod. Phys. A 16 (2001) 3237; D. Kutasov and F. Larsen, J. High Energy Phys. JHEP01(2001)001, hep-th/0009244; F. Lin, Phys. Lett. B 507 (2001) 270; R. Brustein, S. Foffa and G. Veneziano, Phys. Lett. B 507 (2001) 270; D. Klemm, A.C. Petkou and G. Siopsis, Nucl. Phys. B 601 (2001) 380.

[18] D. Bak and S-J Rey, Class. Quantum Grav. 17 (2000) L83.

[19] E. Witten, Science 285 (1999) 512.

[20] P. Horava and D. Minic, Phys. Rev. Lett. 85 (2000) 1610. 
[21] B. Wang, E. Abdalla and T. Osada, Phys. Rev. Lett. 85 (2000) 5507.

[22] T. Banks and W. Fischler astro-ph/0307459; B. Wang and E. Abdalla, Phys. Rev. D 69 (2004) 104014; R.G. Cai, J. Cosmol. Astropart. Phys. JCAP02(2004)007, hep-th/0312014; D.A. Lowe and D. Marolf, Phys. Rev. D 70 (2004) 026001.

[23] M. Li, Phys. Lett. B 603 (2004) 1; Q.G. Huang and Y.G. Gong, J. Cosmol. Astropart. Phys. JCAP08(2004)006, astro-ph/0403590; S.D.H. Hsu, Phys. Lett. B 594 (2004) 13; B. Wang, E. Abdalla and R.K. Su, Phys. Lett. B 611 (2005) 21; Y. S. Myung, Mod. Phys. Lett. A 20 (2005) 2035; Y.G. Gong, B. Wang and Y.Z. Zhang, Phys. Rev. D 72 (2005) 043510; Y.G. Gong and Y.Z. Zhang, Class. Quantum Grav. 22 (2005) 4895.

[24] G.W. Gibbons and S.W. Hawking, Phys. Rev. D 15 (1977) 2738.

[25] M.D. Pollock and T.P. Singh, Class. Quantum Grav. 6 (1989) 901.

[26] A.V. Frolov and L. Kofman, J. Cosmol. Astropart. Phys. JCAP05(2003)009, hep-th/0212327.

[27] B. Wang, Y.G. Gong and E. Abdalla, Phys. Rev. D 74 (2006) 083520.

[28] J.A.S. Lima and A. Maia Jr., Phys. Rev. D 52 (1995) 5628; J.A.S. Lima and J.S. Alcaniz, Phys. Lett. B 600 (2004) 191.

[29] R. Bousso, Phys. Rev. D 71 (2005) 023516.

[30] P.F. González-Díaz and C.L. Sigüenza, Nucl. Phys. B 697 (2004) 363.

[31] I. Brevik, S Nojiri, S.D. Odintsov and L. Vanzo, Phys. Rev. D 70 (2004) 043520; S. Nojiri and S.D. Odintsov, Phys. Rev. D 70 (2004) 103522; B. Guberina, R. Horvat and H. Nikolić, Phys. Lett. B 636 (2006) 80; M.R. Setare and S. Shafei, J. Cosmol. Astropart. Phys. JCAP09(2006)011, gr-qc/0606103.

[32] F.C. Santos, M.L. Bedran and V. Soares, Phys. Lett. B 636 (2006) 86.

[33] H.M. Sadjadi, Phys. Rev. D 73 (2006) 063525; gr-qc/0611114; G. Izquierdo and D. Pavon, Phys. Lett. B. 633 (2006) 420; E. Babichev, V. Dokuchaev and Yu. Eroshenko, Phys. Rev. Lett. 93 (2004) 021102.

[34] A. Kamenshchik, U. Moschella and V. Pasquier, Phys. Lett. B 511 (2001) 265; M. Bento, O. Bertolami and A.A. Sen, Phys. Rev. D 66 (2002) 043507; Y.G. Gong, J. Cosmol. Astropart. Phys. JCAP03(2005)007, astro-ph/0411253.

[35] E. Poisson and W. Israel, Phys. Rev. D 41 (1990) 1796.

[36] C.M. Misner and D.H. Sharp, Phys. Rev 136 (1964) B571.

[37] S.A. Hayward, Phys. Rev. D 53 (1996) 1938; S.A. Hayward, Class. Quantum Grav. 15 (1998) 
3147; S.A. Hayward, S. Mukohyama and M.C. Ashworth, Phys. Lett. A 256 (1999) 347.

[38] J.R. Chisholm, Phys. Rev. D 74 (2006) 043512; T. Harada, Phys. Rev. D 74 (2006) 084004, gr-qc/0609055. 